\title{
Innovative Kunststoffanwendungen für ein kleines Stadtbuskonzept
}

\section{Innovative plastic applications for a small urban bus concept}

Gerhard Kopp, DLR Institut für Fahrzeugkonzepte, Stuttgart

Alexander Müller, Hochschule Esslingen

Oliver Deißer, DLR Institut für Fahrzeugkonzepte, Stuttgart

Stefanie Beyer, Hochschule Esslingen

\section{Kurzfassung / Abstract}

Das Projekt "Reallabor Schorndorf (BOOLEAN)" ist eines von sieben Forschungsprojekten, die gefördert vom baden-württembergischen Ministerium für Wissenschaft, Forschung und Kunst zukunftsfähige Lösungen für Herausforderungen in Ballungsräume erproben. In Reallaboren bearbeiten dabei Wissenschaftler zusammen mit Kommunen, Unternehmen und Bürgern Veränderungen in der Stadt.

Um die besonderen Anforderungen für die zukünftigen, notwendigen Fahrzeugentwicklungen im ÖPNV zu berücksichtigen, wird im Rahmen des vorgestellten Vorhabens unter anderem ein innovatives, leichtes, urbanes Buskonzept entwickelt. In einem mehrstufigen Verfahren werden zuerst Skizzen und Grobentwürfe entwickelt. Diese kommen unter anderem im Rahmen der Bürgerbeteiligung zum Einsatz, um die weiteren Anforderungen an das Fahrzeug zu spezifizieren. In einem nächsten Schritt werden der Entwurf und die Fahrzeugarchitektur konstruktiv umgesetzt.

Eine besondere Herausforderung des Vorgehens liegt bei der Einbindung aller Beteiligten in die Konzeption und Umsetzung des innovativen Fahrzeugkonzepts und dessen technischen Darstellung in einem Fahrzeugmodell, das unter anderem die Themen "Umweltverträglichkeit, Sicherheit und Komfort" adressiert.

Diesen Anforderungen wird insbesondere durch einen systematischen Vergleich von Leichtbaupotentialen ausgewählter Bauweisen begegnet, die unterschiedliche Kunststoffanwendungen und Sandwichstrukturen beinhalten.

Neben der lastpfadoptimierte Rahmenstruktur werden die Hauptstrukturkomponenten Boden, Dach und Seitenwände mit unterschiedlichen Kunststoff- und Sandwichkonfigurationen (SMC, LFT, LFI, ...) ausgelegt und bezüglich Ihres Leichtbaupotentials verglichen. 